\title{
Track-based improvement in the jet transverse momentum resolution for ATLAS
}

\author{
Z. Marshall ${ }^{1,2}$ for the ATLAS Collaboration \\ ${ }^{1}$ Columbia University, Nevis Laboratory, 136 So. Broadway, Irvington, \\ NY 10533, United States of America \\ ${ }^{2}$ California Institute of Technology, Physics Department 103-33, Pasadena, CA 91125, USA \\ Correspondence may be sent to zmarshal@caltech.edu
}

\begin{abstract}
We present a track-based method for improving the jet momentum resolution in ATLAS. Information is added to the reconstructed jet after the standard jet energy scale corrections have been applied. Track-based corrections are implemented, and a $10-15 \%$ improvement in the jet transverse momentum resolution at low $p_{T}$ is achieved. The method is explained, and some validation and physics results are presented. Additional variables are described and analyzed for their resolution improvement potential.
\end{abstract}

\section{Introduction}

ATLAS, one of the general-purpose detectors at the LHC [1], began taking data in 2008. Many of the proposed physics studies include energetic jets, collimated collections of particles often initiated by a single quark or gluon in a hard scatter collision. The calorimeter's response to hadronic jets will be a significant source of systematic uncertainties for both searches for new phenomena, particularly in hadronic decay channels, and Standard Model studies. Understanding the jet energy response is of vital importance. By improving the jet energy resolution, it is possible to improve mass resolution and decrease combinatorial backgrounds as well.

Jet reconstruction in ATLAS has traditionally relied on calorimeter information alone. During event reconstruction, corrections are applied to raw cell energies due to the non-compensating nature of the calorimeter [2]. These jet energy scale (JES) corrections set the energy scale of jets such that -on average- jets have a uniform response in transverse momentum, $R=p_{T}^{\text {reco }} / p_{T}^{\text {true }}=$ 1. However, on a jet-by-jet basis, jets may be over-corrected or under-corrected depending on their topology, particle content, etc.

We describe a technique that uses tracks found within jets to extract information about the jet topology and fragmentation in order to improve the jet transverse momentum resolution [2]. The approach is conceptually different from more traditional energy flow methods [3], where precise track momentum measurements replace calorimeter clusters. Rather, tracks are used to correct the response of jets as a function of the jets' particle composition, using the fraction of

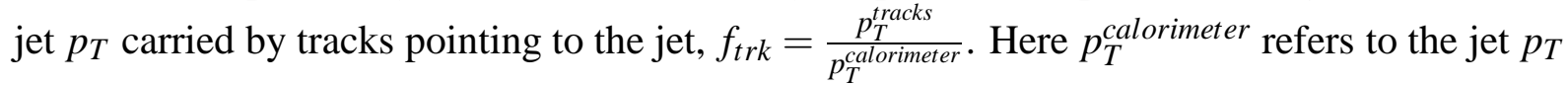



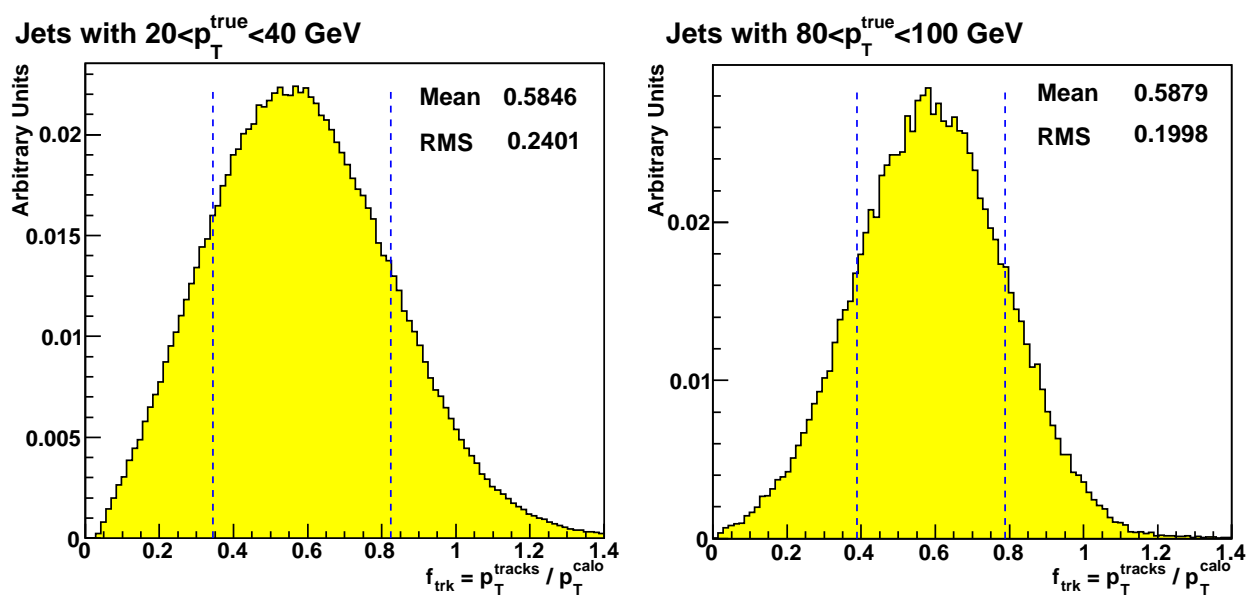

Figure 1: Sample distributions of $f_{t r k}$ for jets in two bins of $p_{T}^{\text {true }}$. Dotted lines are placed at 1 standard deviation above and below the mean of each distribution.

reconstructed using the standard (calorimeter-only) ATLAS reconstruction, and $p_{T}^{\text {tracks }}$ refers to the $p_{T}$ carried by all the tracks found within the jet.

In general, jets are composed primarily of neutral and charged pions. Charged particles leave tracks in the inner detectors, and so one might naively expect that between half and twothirds of the jet $p_{T}$ will be carried by tracks associated with that jet. As shown in Figure 1, Monte Carlo QCD di-jet samples have roughly Gaussian $f_{\text {trk }}$ distributions centered near 0.6, with tails extending above one. The tails are more prominent at low energies and include, for example, jets with a true $f_{\text {trk }}$ near one and one or more tracks with incorrectly measured momenta. In this all-pion approximation of jet composition, the number of tracks corresponds to the hadronic fraction of the jet, and $f_{t r k}$ provides a method for adjusting the jet energy for the non-compensating calorimeter of ATLAS. Additionally, an extreme $f_{\text {trk }}$ can point to a very poorly calibrated jet, since a fluctuation reconstructed jet $p_{T}$ will cause a fluctuation in $f_{\text {trk }}$.

The transverse jet momentum resolution, $\frac{\sigma\left(p_{T}^{\text {reco }}-p_{T}^{\text {true }}\right)}{p_{T}^{\text {true }}}$, where $\sigma$ is the standard deviation of the distribution, is proportional to the width of the jet momentum response in bins of transverse momentum, normalized to the average true jet momentum in a bin. If the response of these jets varies significantly with $f_{t r k}$, the transverse jet momentum resolution will be artificially broadened, as shown in Figure 2. One sees that the total measured transverse jet momentum response distribution is considerably wider than either of the constituent distributions corresponding to jets with different charged particle fractions. By correcting the jet response as a function of jet $p_{T}$ and $f_{t r k}$ we reduce the overall width of the momentum distribution and, hence, improve the jet momentum resolution.

Although the variable $f_{\text {trk }}$ demonstrates the method clearly, this principle holds for other track or calorimeter variables as well. Many variables can hold information about jet topology, including the number of tracks associated to a jet, the spread of the tracks within the jet, and the fraction of track $p_{T}$ carried by the leading track. By using a combination of these variables, one could considerably improve the transverse jet momentum resolution. 

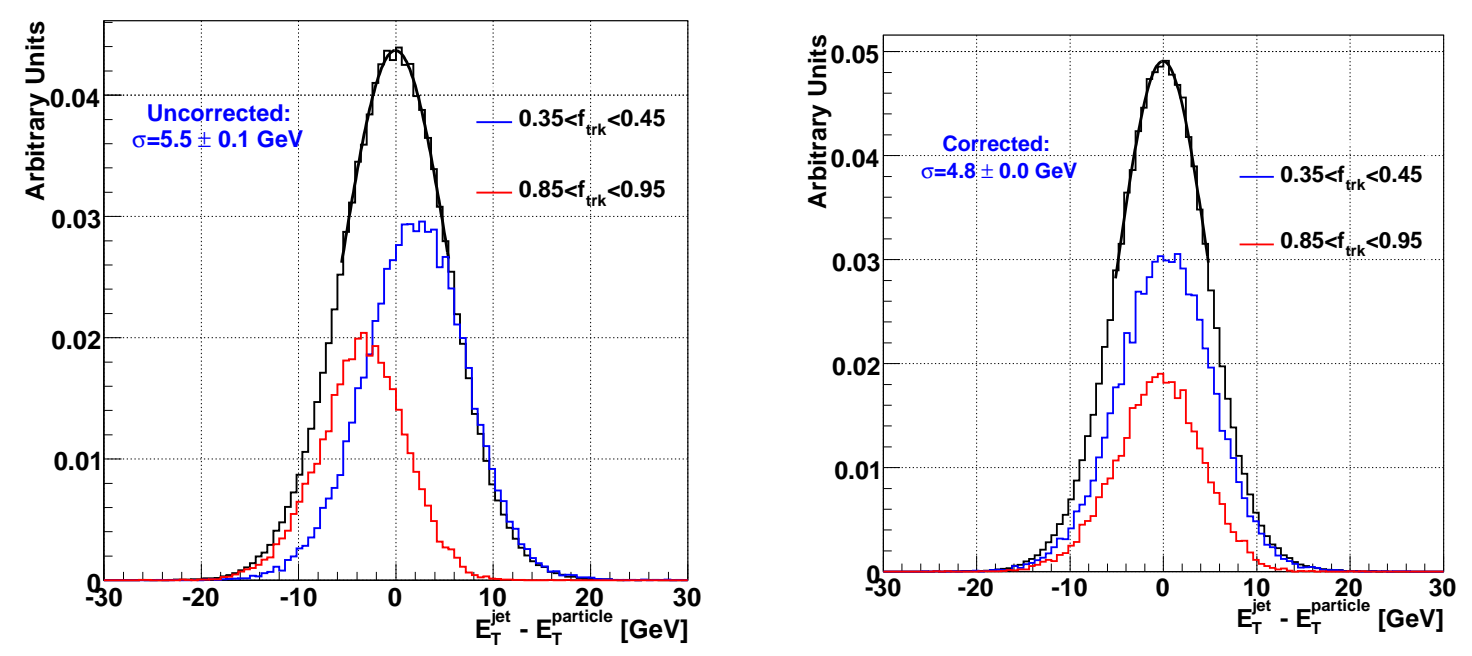

Figure 2: Black: difference between reconstructed and true jet $p_{T}$ for jets with $|\eta|<0.7$ and $40 \mathrm{GeV}<p_{T}<60 \mathrm{GeV}$ before (left) and after (right) a correction based on $f_{\text {trk }}$ is applied. The mean (width) of this distribution is proportional to the jet momentum response (resolution). Since jets with different $f_{t r k}$ have different responses, the transverse momentum resolution is artificially broadened because of the offset of the distributions for each $f_{t r k}$ bin. The $f_{t r k}$ bins are centered at \pm 1 standard deviation in the $f_{\text {trk }}$ distribution. The normalization is arbitrary.

\section{Monte Carlo Samples and Event Selection}

Track-based jet corrections were determined using QCD di-jet events. A list of the samples used, including cross sections and leading parton $p_{T}$ ranges, is included in Table 1. Samples were not normalized.

Table 1: Properties of the QCD di-jet samples used in this analysis. The samples are divided into slices according to the transverse momentum of the leading parton from the hard scatter.

\begin{tabular}{l|l|l|l} 
Sample & Leading Parton $p_{T}$ Range & Cross Section & Number of Events \\
\hline J1 & $17-35 \mathrm{GeV}$ & $1.4 \mathrm{mb}$ & 302,000 \\
J2 & $35-70 \mathrm{GeV}$ & $93 \mu \mathrm{b}$ & $1,161,750$ \\
J3 & $70-140 \mathrm{GeV}$ & $5.9 \mu \mathrm{b}$ & 254,750 \\
J4 & $140-280 \mathrm{GeV}$ & $0.31 \mu \mathrm{b}$ & 336,000 \\
J5 & $280-560 \mathrm{GeV}$ & $12 \mathrm{nb}$ & 321,550 \\
J6 & $560-1120 \mathrm{GeV}$ & $0.4 \mathrm{nb}$ & 371,800
\end{tabular}

Reconstructed tower-seeded, 0.4 cone jets selected from the events ranged in $p_{T}$ from $7 \mathrm{GeV}$ to $1120 \mathrm{GeV}$. Only jets with energies between 20 and $600 \mathrm{GeV}$ were considered to derive the corrections. Fits were formed only for central jets with pseudorapidity $|\eta|<0.7$, however the technique can be extended to other regions of $\eta$. Because the ATLAS tracker acceptance ends at $|\eta|=2.5$, jets beyond $|\eta|=2.0$ must be examined more carefully and may not be suitable 
for this technique. We begin from jets with uniform response, $R=p_{T}^{\text {reco }} / p_{T}^{\text {true }}=1$ on average.

Jets were also required to have a "truth" jet (i.e. a jet made from particles immediately after the hard scatter, prior to any interactions with the detector) matched within 0.1 in $\eta-\phi$ space and were required not to be matched to a truth $c$ - or $b$-quark. Such heavy-flavor jets could have different momentum responses compared to light-quark jets and were excluded from the fits (see Section 4.2). About 3\% of the jets in these di-jet samples were $c$ - or $b$-jets.

Tracks within a cone of radius 0.4 in $\eta-\phi$ around a jet's axis were included in the calculation of $f_{t r k}$. In order to remove jets with a single poorly measured track, jets were required to be associated with at least two tracks to derive the corrections, and each track was required to have $p_{T}>0.5 \mathrm{GeV}$ and $\chi^{2} / D o F<3.0$, both of which are standard ATLAS track quality cuts. No other requirements were placed on the tracks. To determine the track-based momentum corrections, jets were also required to be isolated in a cone of 0.8 in $\eta-\phi$ space, to avoid jet-jet contamination which may skew $f_{\text {trk }}$ considerably for low momentum jets. The removal of this isolation criterion will be explored in future studies.

\section{Determination of the Track-Based Jet Momentum Response Correction}

Jet momentum response is non-Gaussian when plotted in bins of reconstructed transverse momentum but is roughly Gaussian when plotted in bins of true transverse momentum. The momentum response is therefore calculated in bins of true jet momentum. Then, using the definition of jet response, $R \equiv p_{T}^{\text {reco }} / p_{T}^{\text {true }}$, pairs are formed of jet momentum response and reconstructed jet transverse momentum $\left(R\left(p_{T}^{\text {true }}\right), p_{T}^{\text {reco }}\left(p_{T}^{\text {true }}\right)\right)$. These pairs are then used to derive corrections for response. A grid was constructed with 20 bins in $p_{T}$ between 20 and $600 \mathrm{GeV}$, with finer binning at low $p_{T}$, and 12 bins in $f_{t r k}$ between 0 and 1.04 . Grid points were constructed so that each bin had roughly an equal number of jets. Fits to the jet momentum response are made as functions of $f_{\text {trk }}$ and $p_{T}$. A two-dimensional fit was then used to fit the data. Slices of the fit surfaces are shown in Figure 3.

\section{Algorithm Performance}

The track-jet response correction $R\left(p_{T}, f_{t r k}\right)$ described previously was applied to jets selected for determining the parameterization. The jet momentum response was checked as a function of $f_{\text {trk }}$. Figure 3 shows the dependence of the response on $f_{t r k}$ before and after applying the track-based correction. Track-based corrections flatten the response, $R\left(p_{T}, f_{\text {trk }}\right)=p_{T}^{\text {jet }} / p_{T}^{\text {true }}$, to a mean value of unity for all $f_{\text {trk }}<1$. Above $f_{\text {trk }}=1$, the fits do not completely correct the jet momentum response. As the $f_{\text {trk }}$ distribution in Figure 1 demonstrates, very few jets lie in this region, and so the mean transverse momentum resolution is not significantly affected. When correcting jets, a cut was applied at $f_{\text {trk }}=1.4$, above which jets were not corrected owing to inconsistent variation in response.

As described earlier and shown in Figure 2, the jet resolution before the $R\left(p_{T}, f_{t r k}\right)$ correction can be thought of as re-centering several offset Gaussians with different $f_{t r k}$. By recentering the underlying distributions we improve the jet transverse momentum resolution. 

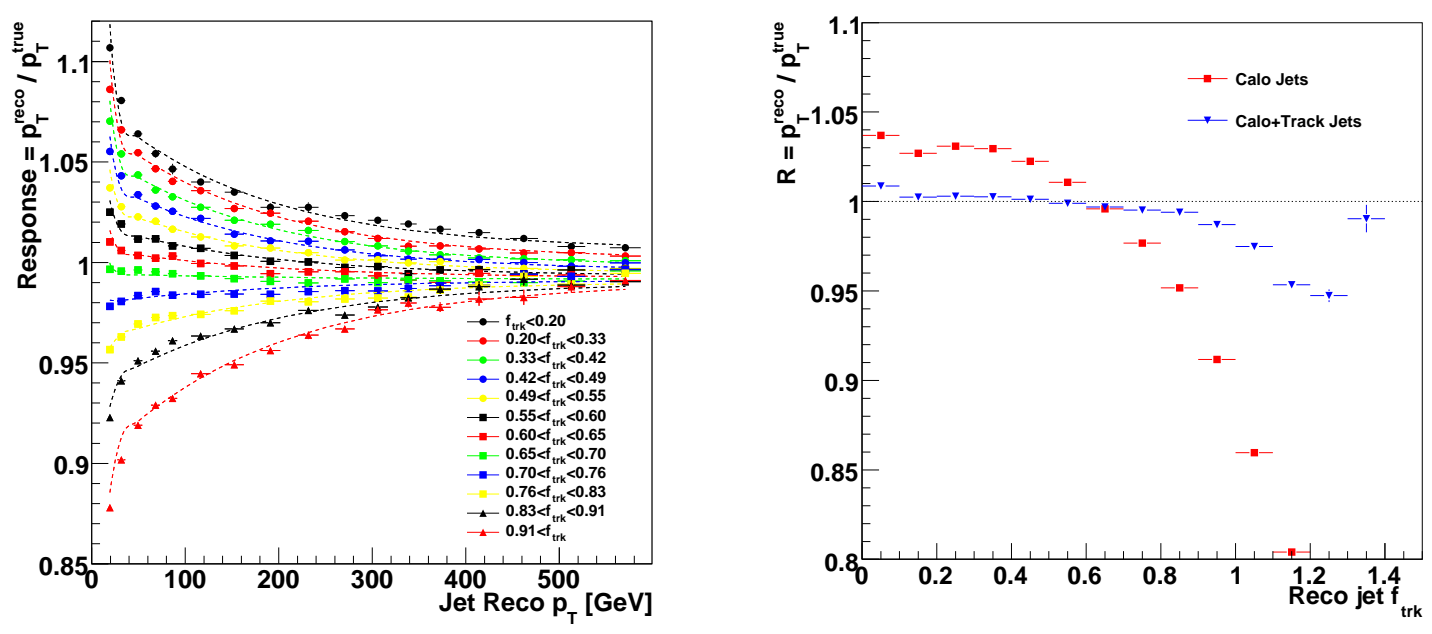

Figure 3: Left, slices of the data and the fit surface. Fits are shown as dotted lines over the data points. Right, jet response as a function of $f_{\text {trk }}$, before (Calo Jets) and after (Calo+Track Jets) applying the track-based response correction. The distribution of $f_{t r k}$, as shown in Figure 1, drops off before one, so few jets are in the poorly corrected high $f_{\text {trk }}$ region.

Figure 2 shows the overlapping distributions after corrections. The tails of the distribution have been improved without affecting the mean.

\subsection{Jet Transverse Momentum Resolution}

The jet transverse momentum resolution is considerably improved at low jet $p_{T}$, as demonstrated in Figure 4. Fits are shown both before and after the corrections are applied. A $10-15 \%$ transverse momentum resolution improvement is achieved at low $p_{T}$. The transverse momentum resolution is fit with a function:

$$
\frac{\sigma\left(p_{T}\right)}{p_{T}}=\sqrt{\frac{a^{2}}{p_{T}}+\frac{b^{2}}{p_{T}^{2}}+c^{2}}
$$

where $a^{2} / p_{T}$ is a stochastic term, $b^{2} / p_{T}^{2}$ is a noise term, and $c^{2}$ is a constant term.

\subsection{Heavy Quark Jets}

Because of presumed differences between the fragmentation of a heavy-quark jet and that of a light-quark jet, there was some concern that their properties would be sufficiently different that the same corrections could not be applied. In fact, this is not the case: $b$-quark tower jet transverse momentum resolution is indeed improved by the same corrections derived from and applied to the light-quark tower jets, and the response is corrected properly. The transverse momentum resolution improvement at $40 \mathrm{GeV}$ is approximately $8 \%$.

A great deal of the concern over heavy-quark jets was caused by the assumption that their distributions for $f_{t r k}$ would be significantly different from those of light-quark jets. The mean 


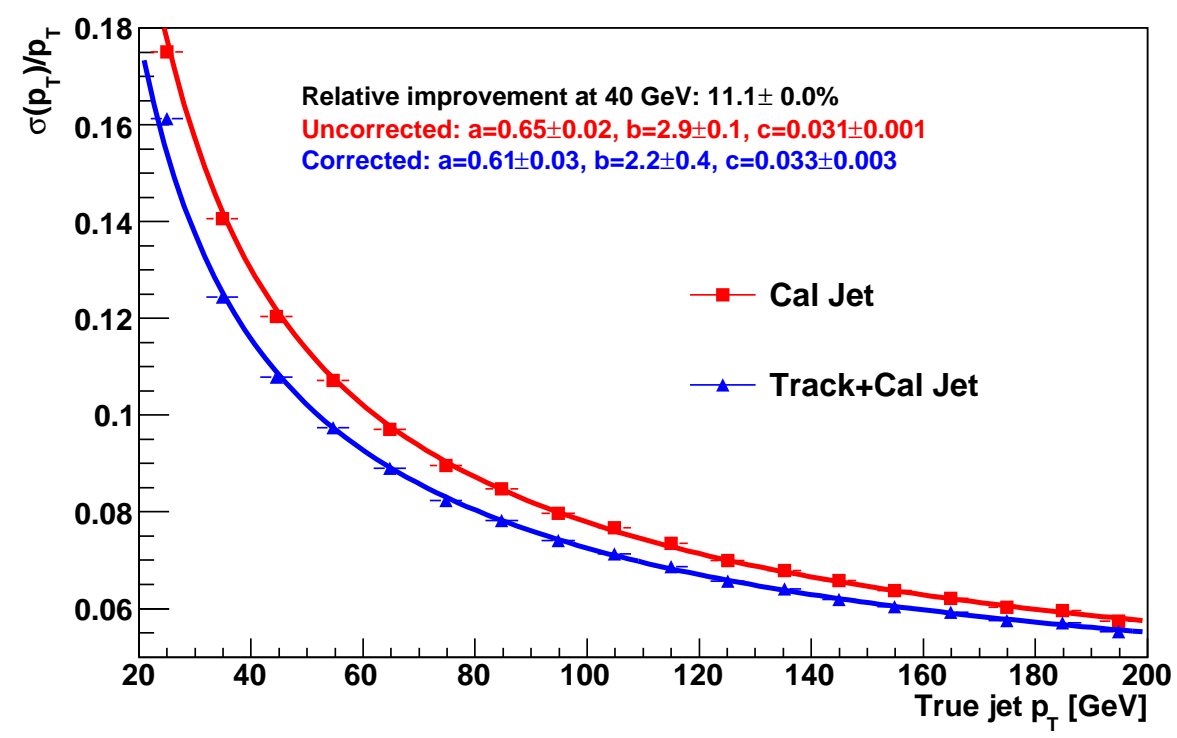

Figure 4: Jet transverse momentum resolution as a function of true jet $p_{T}$ before (Cal Jet) and after (Track+Cal Jet) correcting for $f_{t r k}$.

and standard deviation of each the $f_{t r k}$ and track multiplicity distributions for light- and heavyquark jets can be found in Figure 5. The different types of jets show few significant differences at low $p_{T}$, where this technique is most useful.

\subsection{Missing Transverse Energy Bias}

Track-based jet corrections can also be used to improve the scale of the missing transverse energy, $\mathbb{E}_{T}$. The track-corrected $\mathbb{E}_{T}$ is computed as

$$
\mathbb{E}_{T}^{c o r r}=\mathbb{E}_{T x, y}-\sum_{j e t s}\left(p_{T}^{\text {corr }}-p_{T}^{\text {reco }}\right)
$$

where $p_{T}^{\text {corr }}=p_{T}^{\text {reco }} / R\left(p_{T}^{\text {reco }}, f_{\text {trk }}\right)$ and the sum is over all jets in the event.

Figure 6(a) shows the mean value of the $\mathbb{E}_{T}{ }^{\text {reco }}-\mathbb{E}_{T}{ }^{\text {true }}$ for the J4 sample alone (see Table 1) as a function of the $f_{t r k}$ difference between the two leading jets in a di-jet sample. $\mathbb{E}_{T}{ }^{\text {true }}$ is simply the sum of neutrino $p_{T}$. Both leading jets were required to have $|\eta|<2.0$ and $p_{T}>$ $20 \mathrm{GeV}$ for this study. Events were required to have no more than three reconstructed jets, and the two leading jets were required to have $\Delta \phi>2.9$.

Figure 6(a) shows a large imbalance of transverse energy when the two jets have large differences in $f_{t r k}$. Here $f_{t r k}^{1}\left(f_{t r k}^{2}\right)$ refers to the $f_{t r k}$ of the first (second) leading jet, and the similarly $p_{T}^{1}\left(p_{T}^{2}\right)$ refers to the $p_{T}^{\text {reco }}$ of the first (second) leading jet. When $\Delta f_{t r k}<0, f_{t r k}^{2}>f_{\text {trk }}^{1}$ and $p_{T}^{2}$ is underestimated resulting in a positive bias on $\mathbb{E}_{T}$. A similar argument explains a negative $\not_{T}$ bias for $\Delta f_{t r k}>0$.

Figure 6(b) shows that the $\mathbb{E}_{T}$ scale is properly corrected after applying the track-based response correction to the leading two jets, and the $\mathbb{E}_{T}$ bias has been removed. 
$f_{\text {trk }}$ for different true jet flavors

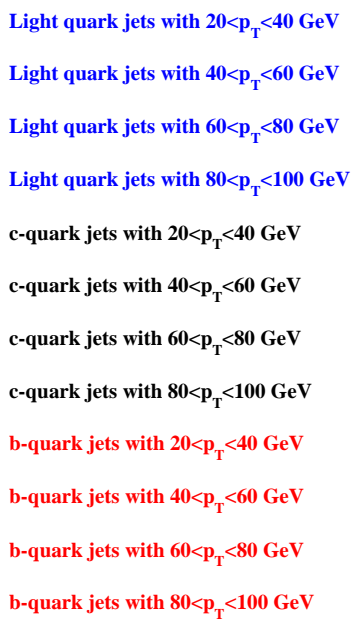

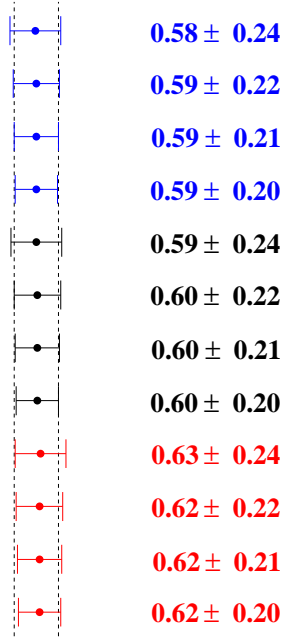

Track Multiplicity for different true jet flavors

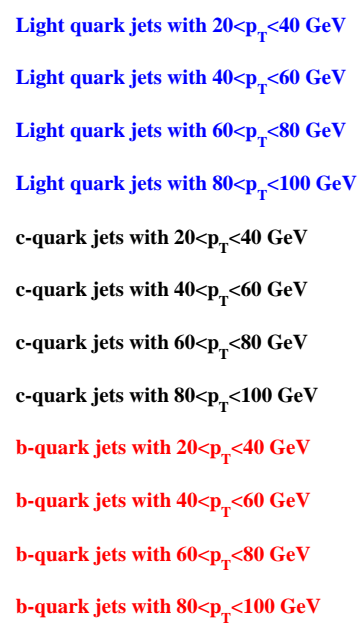

Figure 5: Mean $f_{t r k}$ (left) and track multiplicity (right) in light-quark jets, $c$-quark jets, and $b$ jets. Widths represent the standard deviation of the distribution. Dotted lines are placed at one standard deviation above and below the mean of the $60<p_{T}<80 \mathrm{GeV}$ light-quark distribution.

\section{Evaluation of Additional Variables}

Additional variables must be considered to maximally improve jet transverse momentum resolution. The variables should be uncorrelated in order to not introduce correction ordering problems and should be the minimal set that provides maximum information about jet topology.

The improvement provided by a single variable can be evaluated based on the difference in response between those jets at one standard deviation above and below the mean of the variable distribution. The difference should be related to the improvement in jet transverse momentum resolution that will be seen after a correction has been applied. We evaluate the variables both before and after corrections based on $f_{t r k}$ in order to understand which variable contains the most useful additional information about jet topology.

If $f_{t r k}$ is a measure of the hadronic energy in a jet, the number of tracks pointing to a jet gives some measure of the division of that hadronic energy. A high number of tracks means that hadrons in the jet are each lower energy and may not travel as far into the calorimeter. The softer particles may also result in an increase in non-linearity effects. The variable is highly correlated to jet $p_{T}$ as well. Distributions of track multiplicity are shown in Figure 7, and the variation in response can be seen in Figure 8. Response shows a 3\% variation with track multiplicity. Simply considering the number of tracks pointing to a jet may introduce problems with track reconstruction efficiency, ambiguity between low- and high- $p_{T}$ tracks, and the radius used to associate tracks to jets. Corrections could be explored as a function of a ratio involving track multiplicity in order to resolve some of these issues.

The $p_{T}$ carried by the leading track may also provide a handle on improving the jet transverse momentum resolution. We have examined the ratio of $p_{T}$ of the leading track to the $p_{T}$ carried by all tracks, $p_{T}^{1}$, and the response variation. By using this ratio rather than, for example, 

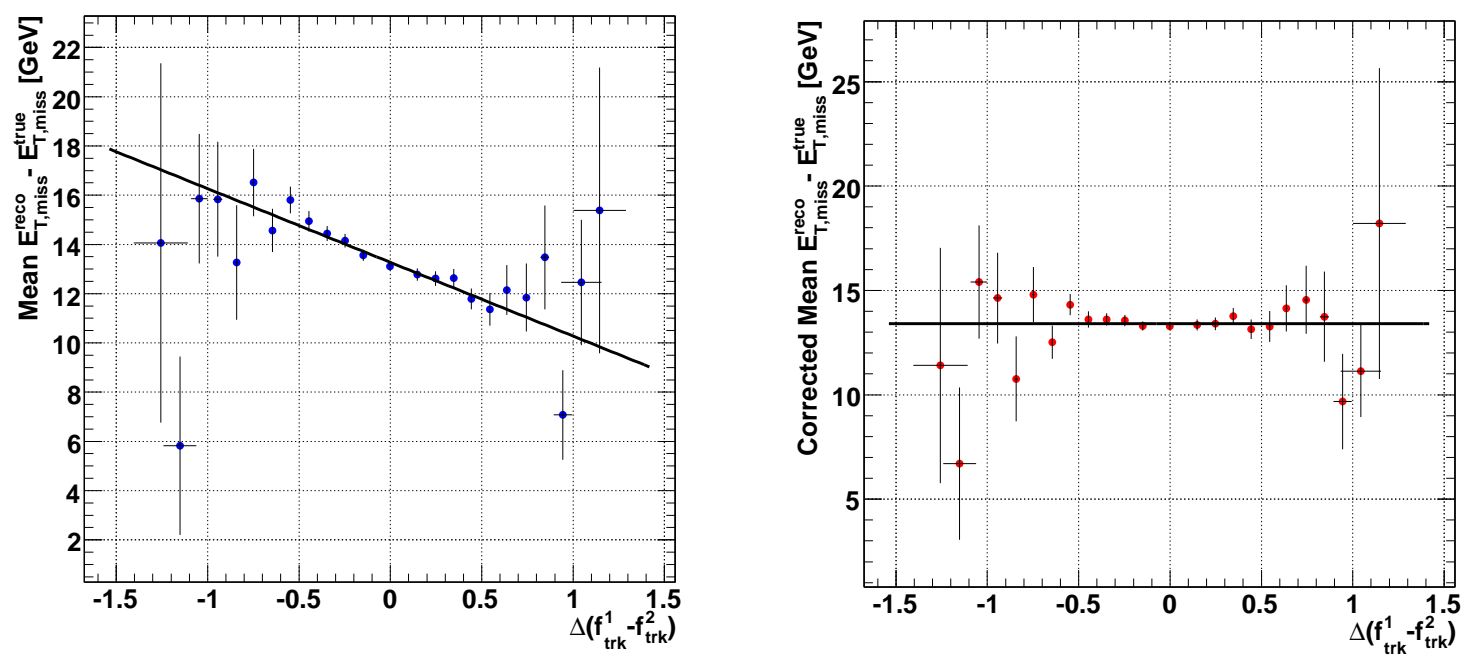

Figure 6: Mean missing transverse energy in a $\mathrm{J} 4$ sample as a function of the $f_{\text {trk }}$ difference between the two leading jets in a di-jet sample before (left) and after (right) track-based jet momentum corrections.

the ratio of $p_{T}$ of the leading track to the $p_{T}$ of the jet, the variable may be less correlated to $f_{\text {trk }}$ and thus independent of corrections based on $f_{t r k}$.

Some measure of track width may also provide useful information about jet topology. A very narrow set of tracks may be included in a single hadronic cluster, whereas several widely spaced tracks may produce several different hadronic clusters. One measure of shower width is the maximum distance in $\eta-\phi$ between any two tracks associated to the jet. One can also construct a measurement of track with using the average distance in $\eta-\phi$ between all tracks, with or without a $p_{T}$ weighting. This variable is somewhat less sensitive to single low- $p_{T}$ tracks on the edges of jets when there is a clear group of high- $p_{T}$ tracks at the core of the jet, a feature that can be particularly important for high- $p_{T}$ jets which typically contain many tracks. Different weighting schemes may also be explored.

Although these variables are each worth exploring, clearly the optimal set will not include all possibilities. The various ways of describing jet width are likely highly correlated, and correction for one may prove sufficient. There are certainly other variables worth exploring that could provide additional information about jet topology either from track information or from some subset of calorimeter information. Initial corrections can be achieved by applying a second fit on top of the $f_{\text {trk }}$ fits already applied. In order to maximize the improvement in response and correctly take into account correlations, full multi-dimensional fits will have to be produced.

\section{Conclusions and Future Studies}

We have introduced a track-based method for correcting the response of jets in ATLAS that provides a $10-15 \%$ improvement in jet transverse momentum resolution at low $p_{T}$ over the 

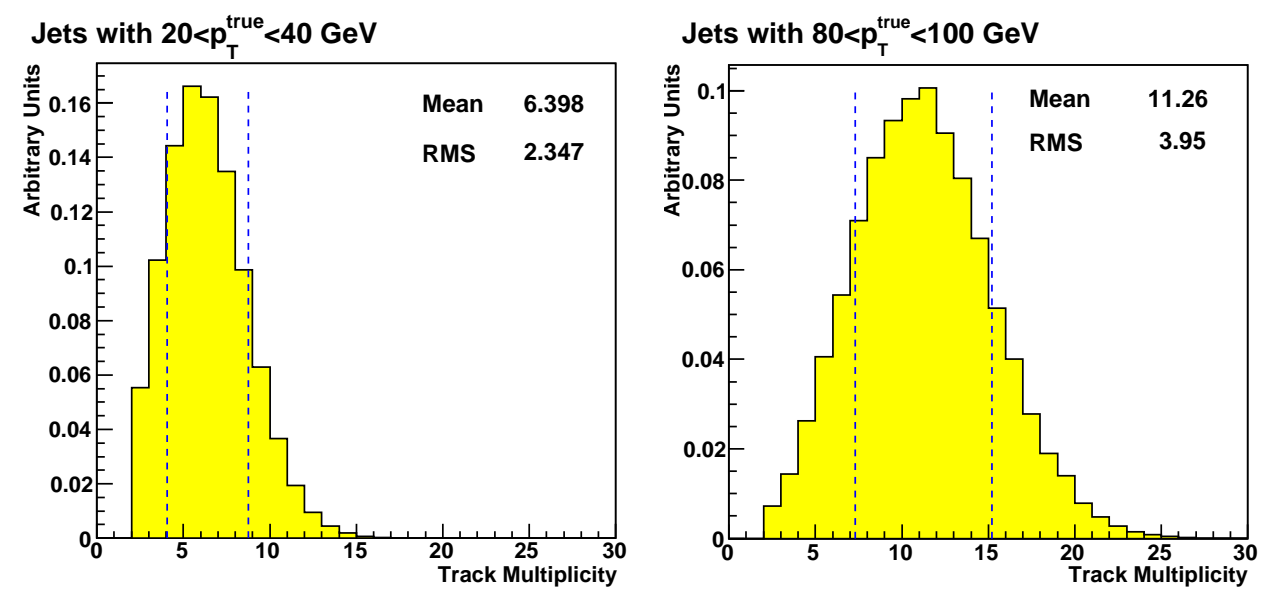

Figure 7: Distributions of track multiplicity for several bins of jet $p_{T}^{\text {true }}$. Dotted lines are placed at one standard deviation above and below the mean of each distribution.

standard ATLAS calorimeter jet reconstruction alone. The corrections derived based on lightquark tower-seeded jets are also effective on $c$ - and $b$-quark jets. The corrections also improve missing energy distributions and biases. These corrections do not require new jet energy scale corrections and can be applied after the standard reconstruction. By systematically adding information from the tracker to jets already reconstructed based on calorimeter information, considerable progress can be made. A tool has been developed in the ATLAS software framework that provides $f_{t r k}$ and a correction based on $f_{t r k}$ as jet moments for all reconstructed jets.

Additional variables have been examined for their ability to improve jet transverse energy resolution. Several variables are promising and will be explored further. Ultimately, some combination of variables will be used to gain the maximum improvement.

These track-based corrections may be sufficiently sensitive to differences in fragmentation between quark- and gluon-initiated jets, for example, that they may be able to account for differences in jet energy scale between the two. By removing the sample-dependence of the jet energy scale corrections, the jet transverse momentum resolution can be improved.

There are several modifications to the technique being explored to further improve the jet transverse momentum resolution using this and other similar track-based variable methods. The radius used for track-jet association may be adjusted to improve performance, and extrapolated track position may be used rather than track direction at the interaction point. Since pile-up will be a significant issue at the LHC even from early on, these corrections will be tested in combination with a jet-vertex association technique already in progress [2].

\section{References}

[1] ATLAS Collaboration, G. Aad et al., JINST3 S08003 (2008).

[2] ATLAS Collaboration, CERN-OPEN-2008-020 (2008).

[3] O. Lobban, A. Sriharan, R. Wigmans, Nucl. Inst. Meth. 495 (2002) 107-120. 

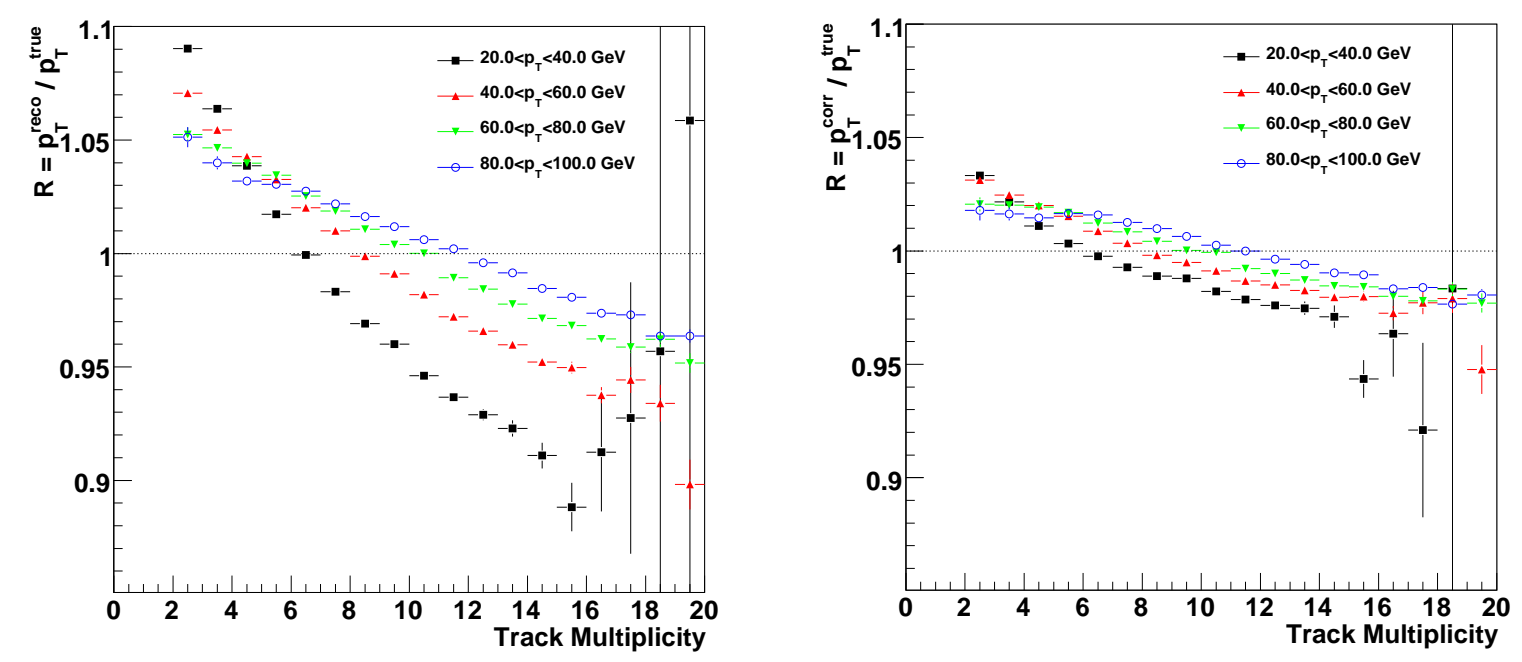

Figure 8: Jet response as a function of track multiplicity, before (left) and after (right) corrections for $f_{t r k}$. A cut required each jet to have at least two tracks associated to it. 